\title{
A focus on quantum dots for luminescent bioanalysis
}

\author{
Alfredo Sanz-Medel • Rosario Pereiro • \\ José Manuel Costa-Fernández
}

Published online: 26 April 2010

(C) Springer-Verlag 2010

The past 20 years have seen a growing research activity in the area of luminescence analysis, mainly because of the high sensitivity and selectivity typically obtained by use of luminescence approaches. As a result, there is a current demand for new luminescent probes for practical chemical and biochemical applications. Thus, the search for improved luminescent indicators is, today, a clear trend in this field. In this direction, the wealth of novel physical, chemical, and biological behaviour of materials on the nanometre scale have brought increasing interest in the synthesis and application of inorganic nanomaterials as an advantageous alternative to conventional organic dyes as luminescent indicators. Particularly unique luminescence emission features can be obtained from inorganic semiconductor nanocrystals, the so-called quantum dots (QDs). Such nanoparticles have varied fascinating optoelectronic properties offering new avenues to develop and construct a new generation of luminescent probes.

Two groundbreaking papers published in Science in 1998, one by the Nie group and the other by the Alivisatos group in the USA, demonstrated that luminescent QDs can be made water-soluble and biocompatible by appropriate surface modification and bioconjugation. This breakthrough boosted new applications of luminescent QDs, particularly in the field of highly sensitive fluorescent biomarkers and (bio)chemical probes. After those pioneering works, and further substantial practical developments, QDs hold special

\footnotetext{
A. Sanz-Medel $(\bowtie) \cdot$ R. Pereiro $\cdot$ J. M. Costa-Fernández Department of Physical and Analytical Chemistry, University of Oviedo,

c/Julián Clavería 8 ,

33006 Oviedo, Spain

e-mail: asm@uniovi.es
}

promise as the next generation of fluorescent probes, and their large impact on fluorescence resonance energy transfer-based bioassays, on labelling of biological cells, and on sensing applications has been widely documented.

No doubt QDs are nowadays already used in many applications. However, some general analytical problems still have to be overcome. A general problem is specificity: in conventional labelling experiments QDs are typically bound to antibodies, specific to the target species or biomolecules in a complex biological sample. Unfortunately well known effects such as nonspecific adsorption still cannot be ruled out. Much effort has been invested in the surface modification of QDs. Another problem hindering their widespread use in the biosciences is that such particles are potentially cytotoxic, etc.

Today, different strategies are being investigated to further extend the application field of such attractive luminescent nanoparticles for analytical and, especially, bioanalytical applications.

The exceptional features of QDs have prompted their use as sensitive and selective fluorescent probes in countless nanoassemblies and in an attempt to overcome limitations of more studied organic and protein-based fluorophores. It is, therefore, not surprising that many reviews, feature articles, and original papers on QDs have appeared in the analytical literature in recent years.

This special issue, containing six papers (four reviews and two original research papers) from universities and research laboratories, is intended to be a valuable addition-a focus on this popular topic of quantum dots as luminescent labels for (bio)chemical, biological, and biomedical purposes. In fact, this selected group of papers provides four critical reviews on particular fields of application of QDs: their use for in-vitro and in-vivo imaging by resorting to detection in either the visible or near infrared region (e.g., the latter review 
highlights the advantageous features of near infrared QDs for deep-tissue imaging). Other interesting strategies, for example the use of host molecule-coated quantum dots as luminescent chemosensors or the development of molecularly imprinted QD nanocomposites for recognition and selective detection of specific proteins (as an alternative to using biochemical recognition) are also discussed. Finally, the last included two original papers report on the use of QDs as valuable molecular probes in practical applications, for example the quantification of the food toxicant methyl parathion and the special recognition of proteins such as amylase, lipase, and lysozyme in saliva for their final accurate determination.

Therefore, we believe that this small collection of papers provides a complementary and updated insight into the growing world of applications of QDs as luminescent labels. Their reading is recommended to experts in the topic and to anyone who wishes to obtain a more detailed picture of the symbiosis between quantum dots, bioconjugation, and analytical/bioanalytical applications.

Let us conclude this short editorial by thanking all the authors who contributed to this special issue, the referees of the papers for their thorough and timely reviews, and, last but not least, to the $\mathrm{ABC}$ editorial staff for their guidance.

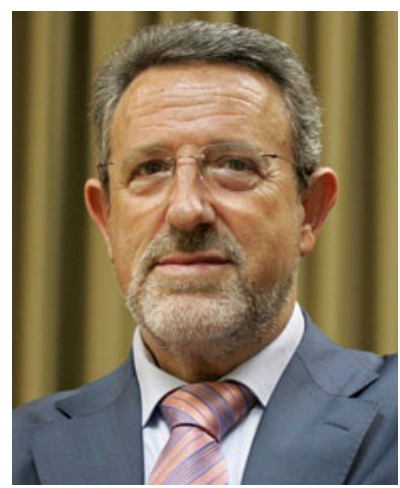

Alfredo Sanz-Medel

has been Professor in the Department of Physical and Analytical Chemistry of Oviedo University (Spain) since 1982. He is author or co-author of approximately 500 scientific publications in international journals, several patents, and books. His current research interests include new atomic detectors and ion sources for ultra-trace analysis using plasmas, new molecular optical sensors, particularly those based on the use of quantum dots, and hybrid techniques, coupling a separation unit and an atomic detector, for ultra-trace and trace metal speciation to solve biological and environmental problems and speciation for proteomics. This is aimed at by integrating mass spectrometry (MS) "molecular" (matrix-assisted laser desorption/ionization and electrospray MS), and "atomic" (inductively coupled plasma (ICP)MS) techniques, and introducing the extensive use of ICP-MS to carry out "heteroatom-tagged proteomics", both for qualitative and quantitative purposes. He has been an editor of Analytical and Bioanalytical Chemistry since 2002. At Euroanalysis 2007, in Antwerp, he received the Robert Kellner Award.

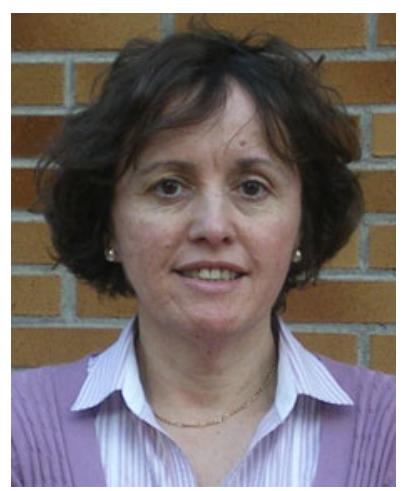

\section{Rosario Pereiro}

is an Associate Professor at the University of Oviedo (Spain) and was recently accredited as Full Professor in Analytical Chemistry. Her current research interests include investigation of new strategies to improve selectivity and/or sensitivity in molecular luminescence by using nanostructured sensing phases (e.g. molecular-imprinted polymers, quantum dots), and the development of analytical strategies for in-depth profile quantitative analysis of thin layers in advanced materials using optical emission and mass spectrometry. She has co-authored more than 100 scientific papers and several patents.

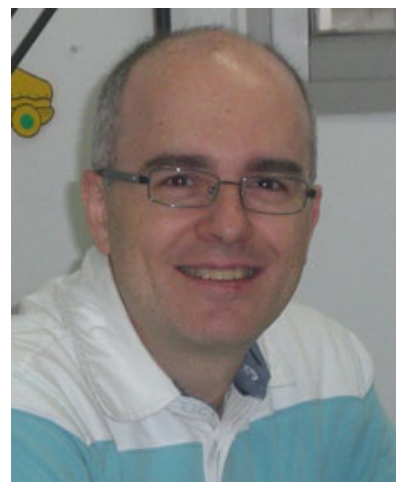

José Manuel Costa-Fernández is an Associate Professor at the Department of Physical and Analytical Chemistry and ViceDean of the Chemistry Faculty at the University of Oviedo (Spain). His current research interests include the development of functionalized photoluminescent nanomaterials (e.g. quantum dots and molecularly imprinted polymers) for biolabelling and sensing applications and the use of plasma-based elemental mass spectrometry for direct analysis of solids and for chemical speciation studies. He is co-author of more than 70 papers in international scientific journals, two patents, and chapters in several books, and co-supervises several $\mathrm{PhD}$ students. 